\title{
Interferometric Observations of Chemistry in High-Mass Star-Forming Regions
}

\author{
Peter Schilke and Karl M. Menten \\ Max-Planck-Institut für Radioastronomie, Auf dem Hügel 69, 53121 \\ Bonn, Germany \\ Friedrich Wyrowski \\ Department of Astronomy, University of Maryland, College Park, MD \\ 20742-2421, USA \\ C. M. Walmsley \\ Osservatorio Astrofisico di Arcetri, Largo E.Fermi 5, I-50125 Firenze, \\ Italy
}

\begin{abstract}
Single dish spectral line surveys of high mass star-forming regions provide spectra with a very high line density, and reveal the presence of many complex molecules. Besides the prototypical Orion BN/KL region, more and more regions get surveyed and we start to get a better idea of the chemical similarities and differences. Yet, single dish studies miss an important aspect of hot cores, which is revealed by higher resolution studies with interferometers: the cores are not chemically homogeneous, but a pronounced chemical substructure exists. As an example of such an interferometric study, we will present one particular set of objects, the UC HII W3 $(\mathrm{OH})$ and its neighboring hot core $\mathrm{W} 3\left(\mathrm{H}_{2} \mathrm{O}\right)$ (otherwise known as the Turner-Welch object), and discuss their chemical properties.
\end{abstract}

\section{Introduction}

High mass stars, although few in number, have a profound influence on their galactic environment through their outflows, UV radiation, stellar winds and eventually, their explosion as a supernova. In contrast to low mass stars, little is known about the formation process of high mass stars, partly because they spend a considerable fraction of their lifetime embedded in their maternal cloud, and thus become optically visible only at a well advanced stage of development. A transition stage between the protostellar and the stellar evolution stage is the hot core phase, in which the emerging star has not yet started to ionize its surroundings, forming a UC HII region, but already has enough energy output to heat the gas to temperatures of typically $100-250 \mathrm{~K}$. This temperature is elevated enough to evaporate any ice mantles that have formed during the cold collapse phase, liberating many molecular species. Since the gas is hot and dense 


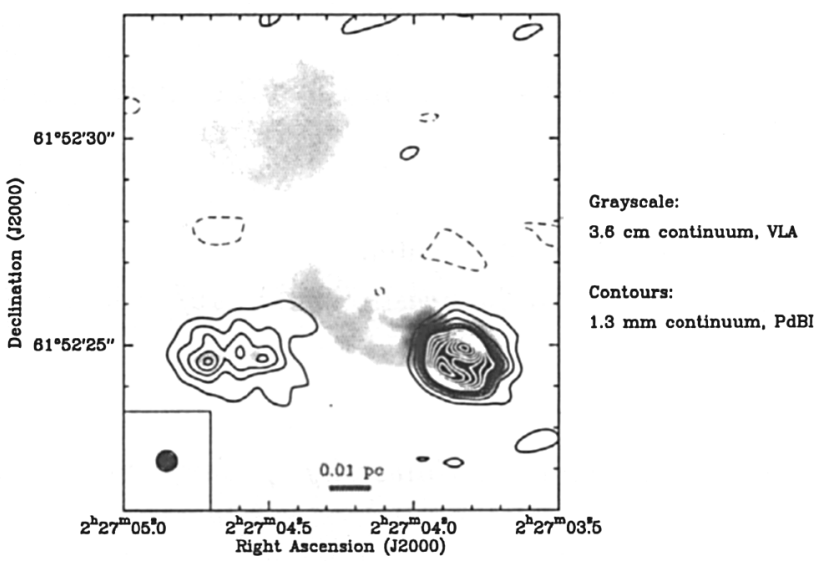

Figure 1. Maps of the $\mathrm{W} 3(\mathrm{OH})$ region at $3.6 \mathrm{~cm}$ with the VLA (Wilner, Reid, \& Menten 1999) [grayscale], and at $1.3 \mathrm{~mm}$ with the Plateau de Bure (Wyrowski et al. 1999) [contours] with 0.8" resolution. The western source is the UC HII region W3 $(\mathrm{OH})$, the eastern sources comprise the hot core $\mathrm{W} 3\left(\mathrm{H}_{2} \mathrm{O}\right)$. The VLA traces mostly freefree continuum emission, with the exception of the eastern source of W3 $\left(\mathrm{H}_{2} \mathrm{O}\right)$, which turns out to have a synchrotron jet. The PdBI traces also free-free emission toward $\mathrm{W} 3(\mathrm{OH})$, but dust continuum emission toward $\mathrm{W} 3\left(\mathrm{H}_{2} \mathrm{O}\right)$.

enough to excite many molecular transitions, one characteristic of hot cores is a very rich millimeter- and submillimeter molecular spectrum.

The molecules observed are a mixture of unprocessed cold cloud material stored on ice mantles, grain surface chemistry products, and the result of hot gas phase chemistry. Observations of the chemical composition are a snapshot of this evolving mixture. Determining abundances and comparing with models can then unravel the history of star formation in various ways:

- the distribution of unprocessed molecules reflect the structure of the collapsing cloud prior to star formation,

- the abundances of molecules produced by grain surface chemistry are determined by the physical conditions in the dense core during star formation,

- abundance ratios of evaporated mother and gas phase daughter species provide a chemical clock to determine how much time has passed since the grain mantles evaporated, i.e. since the star switched on and started to heat its surroundings.

Of particular interest is the spatial distribution of the various tracers, since only that allows to determine the spatial structure of star formation.

The only way to get this information is through interferometric observations, since most massive star forming cores are too far away to be resolvable by single dish telescopes. Yet, interferometric observations of chemistry in high 
mass star forming regions are scarce, with the notable exception of Orion. This region was found to consist of various sub-sources (hot core, ridge, compact ridge: Blake et al. 1987, 1996; Wright, Plambeck, \& Wilner 1996), and different molecules have different spatial distributions. Some molecules or rather families of molecules peak toward the hot core (e.g. nitrogen bearing species), other families prefer the compact ridge (e.g. oxygen bearing species) and some families live in the ridge region (e.g. radicals) (see e.g. Wilner, Plambeck, \& Wright 1996 or Blake et al. 1996). Chemical models have been constructed which try to explain this difference in terms of the thermal history of the region (Caselli, Hasegawa, $\&$ Herbst 1993). From the results obtained in Orion we learn that high mass star-forming clouds do have distinctive chemical substructure and that trying to understand the chemistry of these regions based on single dish results can be perilious, because they are lumped together in a large beam. High spatial resolution seems mandatory.

\section{Need for High Resolution Observations}

Most high mass star-forming regions are so far away that it is very difficult with present day millimeter interferometers to achieve the spatial resolution needed for detailed analyses of their chemistry. Hot cores have diameters in the range 0.01 to $0.05 \mathrm{pc}$, which translate to angular diameters of $4^{\prime \prime}$ to $20^{\prime \prime}$ at the Orion distance of $450 \mathrm{pc}$. Experience with the latter source clearly indicates that higher resolution is needed to resolve chemically different sub-structures. But already one of the next closest hot cores [associated with the ultracompact Hil region W3 $(\mathrm{OH})]$ is four times farther away and has a size of $3^{\prime \prime}$ only. Resolving this core, as we shall see, is possible with current millimeter interferometers, which achieve resolutions in the $1^{\prime \prime}$ range. More typical distances are 5-10 kpc, and the spatial resolutions needed there, with the required sensitivities, are not available today ${ }^{1}$ - except with the VLA, but this instrument allows observations of only a limited number of molecules (e.g. $\mathrm{NH}_{3}$ ), due to its frequency coverage. Another obstacle for chemical studies at the present-day VLA are the very limited spectrometer capabilities, although this will change with the planned upgrade of the VLA. Yet, observations of chemistry in objects other than Orion and $\mathrm{W} 3\left(\mathrm{H}_{2} \mathrm{O}\right)$ are necessary, because one wants to

- distinguish between common traits and individual features,

- trace a number of objects in various stages of evolution,

- trace a number of objects with different masses - both Orion and $\mathrm{W} 3\left(\mathrm{H}_{2} \mathrm{O}\right)$ have around $10 \mathrm{M}_{\odot}$, while other objects such as G10.47+0.03 have about 100 times as much!

The Atacama Large Millimeter Array (ALMA) will allow observations of molecular hot cores throughout the Galaxy at more than ten times better resolution than today and with excellent brightness sensitivity.

\footnotetext{
${ }^{1}$ Some particularly large hot cores [SgrB2(N), Liu \& Snyder 1999] can be observed with current instruments, and BIMA in its extended configuration can achieve $0.5^{\prime \prime}$ resolution, but with low brightness sensitivity.
} 


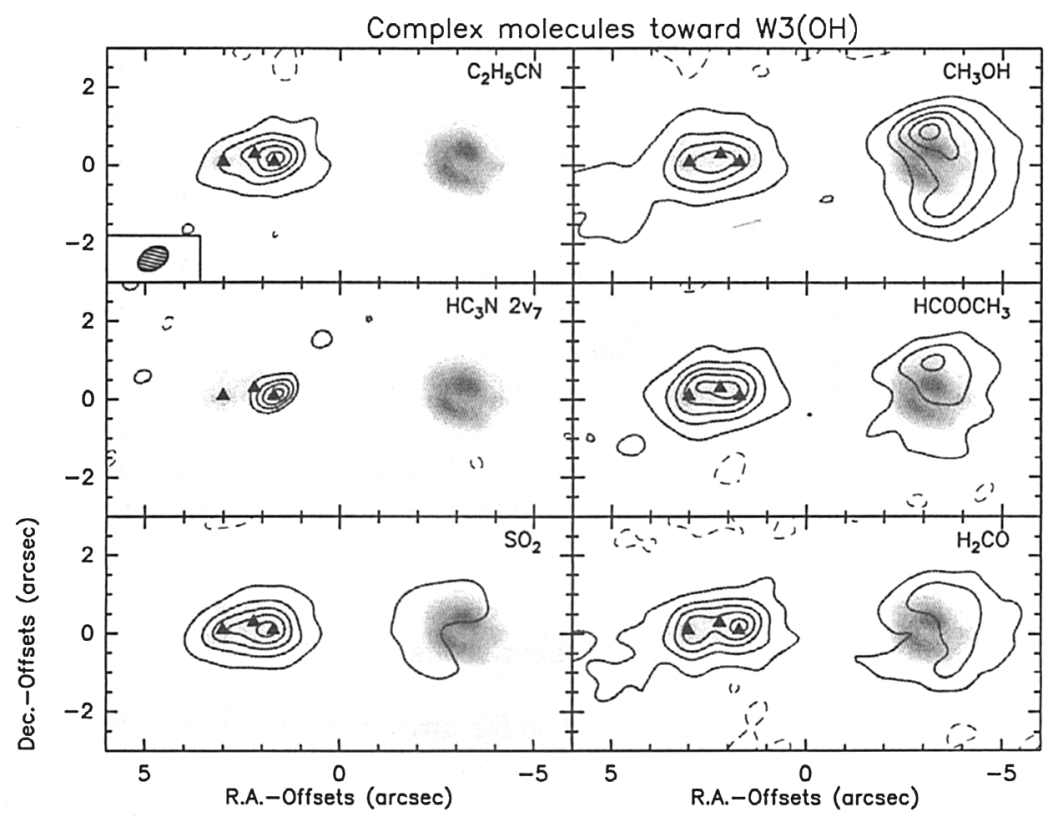

Figure 2. Line observations of $\mathrm{W} 3(\mathrm{OH})$ with the Plateau de Bure Interferometer with $0.8^{\prime \prime}$ resolution. In gray scale the $1.3 \mathrm{~mm}$ dust emission is shown, with the UC HII W3 $(\mathrm{OH})$ in the west and the hot core $\mathrm{W} 3\left(\mathrm{H}_{2} \mathrm{O}\right)$ in the east. The dust emission is triple peaked, the peaks are indicated by triangles. Superimposed on the dust emission are various line maps, which are labeled in the upper right corner of the individual panels.

\section{An Example of What Can Be Done Today: W3(OH)/W3( $\left(\mathrm{H}_{2} \mathrm{O}\right)$}

As an example of what can be achieved with today's instrumentation, observations conducted with the IRAM Plateau de Bure Interferometer (PdBI) toward the hot core $\mathrm{W} 3\left(\mathrm{H}_{2} \mathrm{O}\right)$ and the adjacent ultracompact HII region $\mathrm{W} 3(\mathrm{OH})$ are presented. The W3 $\left(\mathrm{H}_{2} \mathrm{O}\right)$ hot core has a mass of $\approx 15 \mathrm{M}_{\odot}$ and a luminosity of $\approx 5 \times 10^{4} \mathrm{~L}_{\odot}$ (Wyrowski et al. 1999), which is similar to the Orion-KL hot core. The $\mathrm{W} 3\left(\mathrm{H}_{2} \mathrm{O}\right)$ region is one of the closest hot cores $(2 \mathrm{kpc})$ after Orion-KL. The object was observed simultaneously at $3 \mathrm{~mm}$ and $1.3 \mathrm{~mm}$, both in continuum and a multitude of lines (Figs. 1,2; see Wyrowski et al. 1999 for a detailed account of the observations).

The sub-arcsec resolution continuum observations at $1.3 \mathrm{~mm}$ resolved the hot core into three apparent sub-sources (Fig. 1), which are aligned both with the axis defined by $\mathrm{W} 3(\mathrm{OH})$ and $\mathrm{W} 3\left(\mathrm{H}_{2} \mathrm{O}\right)$ and with the water maser outflow originating in one of them (Alcolea et al. 1992). Two of those (the outer ones) also show up in cm maps with the VLA (Reid et al. 1995; Wilner et al. 1999). A synchrotron jet and a water maser outflow are centered on the eastern clump, while the western clump has a thermal $\mathrm{cm}$ spectrum. The distribution of the 
HNCO maps and temperatures toward $\mathrm{W} 3\left(\mathrm{H}_{2} \mathrm{O}\right)$
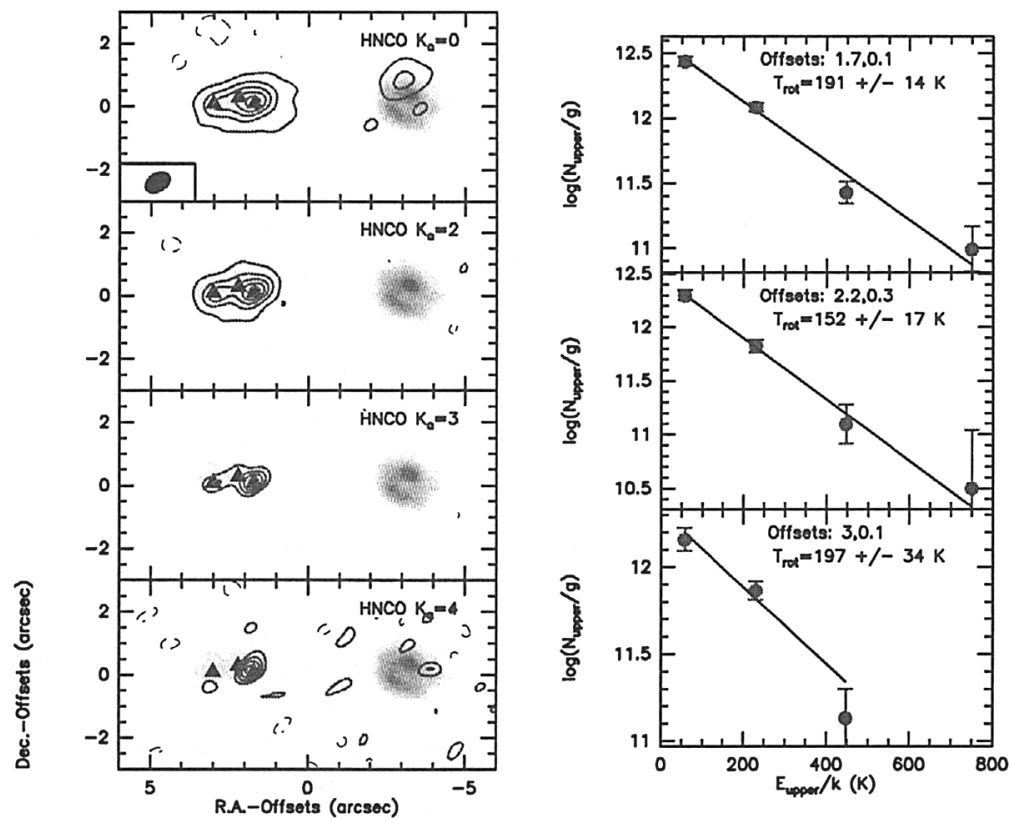

Figure 3. Maps of different $K$-ladder transitions from HNCO (left) and rotation diagrams toward the three continuum peaks (right).

molecules is not uniform: while oxygen bearing molecules are seen all over the core, nitrogen bearing and sulphur bearing species cluster toward the western peak.

The W3(OH) UC HII region is close by and allows studies of a later stage in the evolution of young stellar objects. The gas in the immediate vicinity of the star has been ionized, but the dense molecular shell around it can still be observed in a number of molecules. Again, a distinct chemical signature is seen: only oxygen bearing molecules are observed there, but the detected species include complex organic molecules. Those are found toward the north-western and western edge of the UCHII, while the eastern side is only visible in sulphur bearing molecules.

The maps shown in Fig. 2 suggest distinct chemical differences between different sub-regions, but one has to carefully disentangle excitation and abundance effects to really locate chemical differences. In the present case, multi-line observations of $\mathrm{HNCO}$ enabled us to produce a temperature map of $\mathrm{W} 3\left(\mathrm{H}_{2} \mathrm{O}\right)$. Since all the observed lines were measured simultaneously, we have a very accurate relative calibration. The temperature was determined using a rotation diagram fit (Fig. 3). The different $K$-levels of HNCO used here are thought to be mostly radiatively pumped by FIR radiation (Churchwell et al. 1986). In this case, we can safely assume both thermalization of the lines, and equal kinetic and FIR radiation temperatures; so the temperature we determine should be a close approximation to the kinetic temperature, bearing in mind that our observations yield line-of-sight averages. 


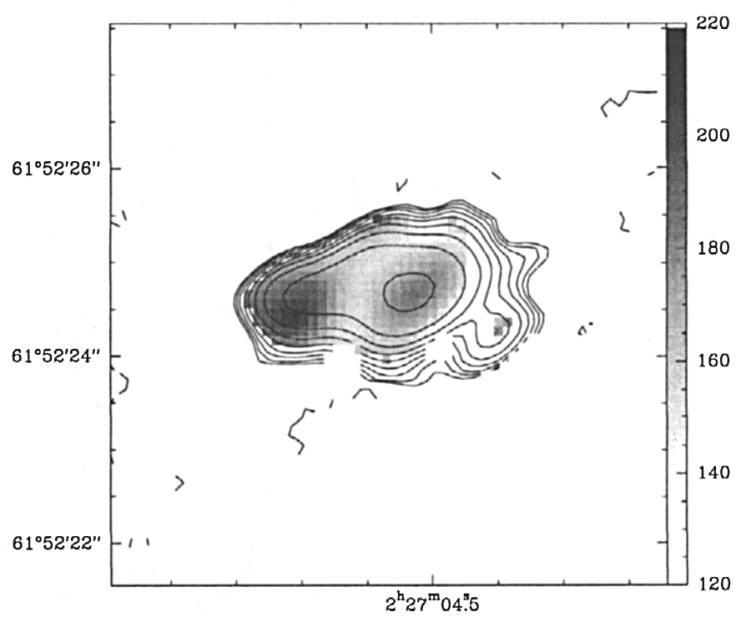

Figure 4. Kinetic temperature map of the $\mathrm{W} 3\left(\mathrm{H}_{2} \mathrm{O}\right)$ source, as derived from the HNCO data. The scale (in Kelvin) is given on the wedge. To ease comparison, contours of the $1.3 \mathrm{~mm}$ continuum (Fig. 1) are shown as well. The central continuum peak does not correspond to a temperature maximum, but the eastern and western peaks (which also contain VLA sources) do.

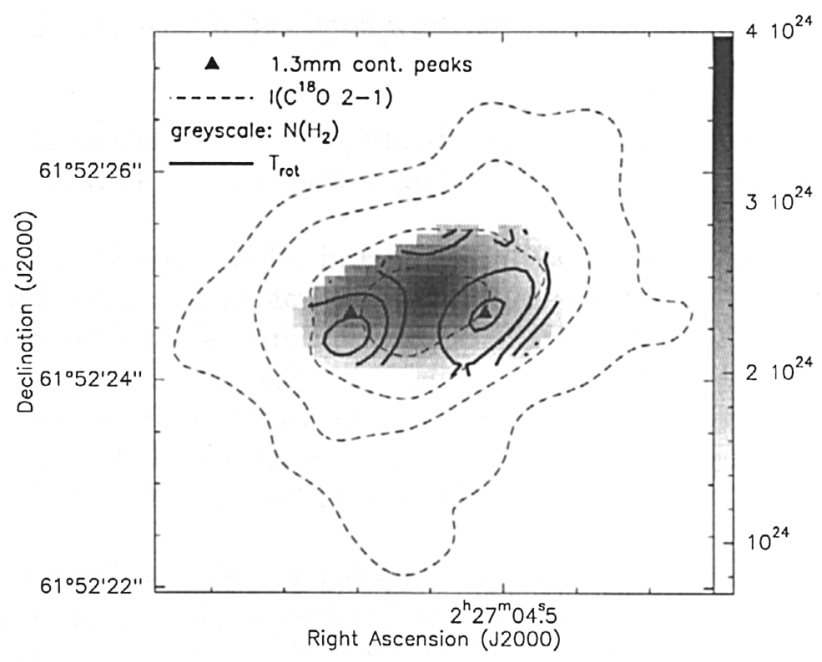

Figure 5. Map of the $\mathrm{H}_{2}$ column density in $\mathrm{W} 3\left(\mathrm{H}_{2} \mathrm{O}\right)$, as derived from the $1.3 \mathrm{~mm}$ continuum data, and using the temperature distribution shown in Fig. 4 [grayscale]. It appears to be single peaked. The three continuum peaks are indicated as triangles, and the temperature map is overlaid as thick contours. The $\mathrm{C}^{18} \mathrm{O}(2-1)$ integrated intensity map, which also traces column density, is drawn as dashed contours. It also appears single peaked, therefore confirming the results obtained from the continuum. 


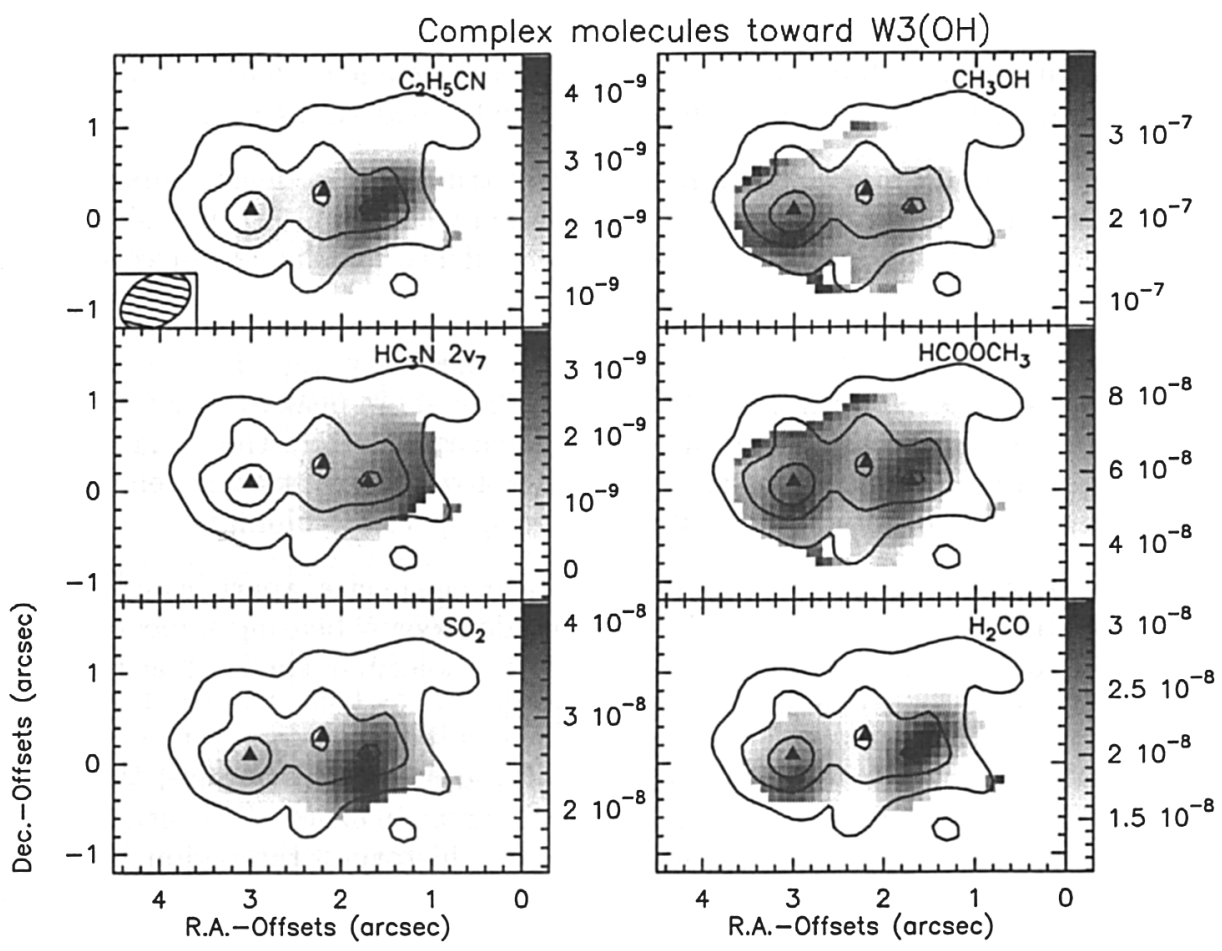

Figure 6. Maps of abundances toward $\mathrm{W} 3\left(\mathrm{H}_{2} \mathrm{O}\right)$, obtained using the maps in Fig. 2, the temperature distribution in Fig. 4 and the $\mathrm{H}_{2}$ column density from Fig. 5. To guide the eye, the $1.3 \mathrm{~mm}$ continuum map has been overlaid as contours. The oxygen bearing species [right panels] have a uniform distribution somewhat centered toward both temperature peaks, while the nitrogen and sulphur bearing species are seen almost exclusively toward the western peak.

Using the temperature map (Fig. 4), we subsequently produced an $\mathrm{H}_{2}$ column density map, based on the $1.3 \mathrm{~mm}$ dust continuum (Fig. 5) and using standard assumptions about dust properties. A check on the validity of this approach was offered by comparison with a $\mathrm{C}^{18} \mathrm{O}(2-1)$ map, which was observed at the same time. The intensity of the $\mathrm{C}^{18} \mathrm{O}(2-1)$ line is not very temperature sensitive and hence traces the column density fairly accurately. The distributions of column density derived from the continuum data and the $\mathrm{C}^{18} \mathrm{O}(2-1)$ line map show good agreement.

Once more using the temperature map, we derived column density maps for various molecules and finally, with the help of the $\mathrm{H}_{2}$ column density map, we generated abundance maps (Fig. 6).

We can summarize the results of our millimeter interferometric study of $\mathrm{W} 3(\mathrm{OH}) / \mathrm{W} 3\left(\mathrm{H}_{2} \mathrm{O}\right)$ as follows: 
- Of the three peaks visible in the $1.3 \mathrm{~mm}$ continuum map, two are temperature peaks. The positions of these peaks coincide with VLA $3.6 \mathrm{~cm}$ sources, strongly suggesting heating by embedded young stars.

- One of the $1.3 \mathrm{~mm}$ continuum peaks is a true column density maximum. This conclusion is confirmed by comparison with the $\mathrm{C}^{18} \mathrm{O}$ intensity map, which only has a weak temperature dependence and hence traces mostly column density.

- The abundances of oxygen-bearing molecules vary very little (within a factor of 2 or 3 ) within the hot core. They show peaks toward the two embedded sources. Nitrogen-bearing species, on the other hand, show larger abundance contrasts. They peak toward the western embedded source. The distribution of sulphur bearing species is similar.

- Toward the UCHII region, no nitrogen bearing species, apart from HNCO, have been found, but a number of organic oxygen bearing molecules are seen toward the north-eastern part of its molecular shell. The western shell is only seen in sulphur bearing species.

Many of the above characteristics of $\mathrm{W} 3\left(\mathrm{H}_{2} \mathrm{O}\right)$ and the shell around $\mathrm{W} 3(\mathrm{OH})$ are reminiscent of the hot core/compact ridge sources in Orion. The situation in Orion has been interpreted as due to the thermal history of the region (Caselli, Hasegawa, \& Herbst 1993) during the collapse phase, where the compact ridge belonged to an outer subshell with a lower temperature than the hot core. Similarly, the dense gas around $\mathrm{W} 3(\mathrm{OH})$ is an outer shell of the core forming the star in the center of the UC HII and features a high abundance of oxygen-bearing and an absence of nitrogen-bearing species, just like the compact ridge in Orion. The W3 $\left(\mathrm{H}_{2} \mathrm{O}\right)$ and Orion hot cores contain both nitrogen and oxygen-bearing molecules. There are differences as well: while the hot core and compact ridge in Orion are subsources of the same star forming core, W3 $(\mathrm{OH})$ and $\mathrm{W} 3\left(\mathrm{H}_{2} \mathrm{O}\right)$ are separate star forming cores, and there is no real equivalent in Orion of the clear chemical dichotomy within $\mathrm{W} 3\left(\mathrm{H}_{2} \mathrm{O}\right)$.

To summarize, the situation in Orion is by no means exceptional, and one has to expect spatial chemical variations in other hot cores as well. With present day millimeter-interferometers it is difficult to have sufficient spatial resolution for other hot core regions which are generally more distant, but ALMA will shed more light on this phenomenon in a larger sample of hot cores.

\section{References}

Alcolea, J., Menten, K.M., Moran, J.M., \& Reid, M.J. 1992 in Astrophysical Masers, eds. A.W. Clegg \& G. Nedoluha (Springer)

Blake, G.A., Mundy, L.G., Carlstrom, J.E., Padin, S., Scott, S.L., Scoville, N.Z., \& Woody, D.P. 1996, ApJ, 472, L49

Blake, G.A., Sutton, E.C., Masson, C.R., \& Phillips, T.G. 1987, ApJ, 315, 621

Caselli, P., Hasegawa, T.I., \& Herbst, E. 1993, ApJ, 408, 548

Churchwell, E., Wood, D., Myers, P.C., \& Myers, R.V. 1986, ApJ, 305, 405

Reid, M.J., Argon, A.L., Masson, C.R., Menten, K.M., \& Moran, J.M. 1995, ApJ, 443, 238 
Wilner, D.J., Reid, M.J., Menten, K.M. 1999, ApJ, 513, 775

Wright, M.C.H., Plambeck, R.L., \& Wilner, D.J. 1996 ApJ, 469, 216

Wyrowski, F., Schilke, P., Walmsley, C.M., \& Menten, K.M. 1999, ApJ, 514, L43

\section{Discussion}

E.F. van Dishoeck: Do you have any explanation for the variations in the abundances of the oxygen- and nitrogen-bearing molecules between $\mathrm{W} 3\left(\mathrm{H}_{2} \mathrm{O}\right)$ and $\mathrm{W} 3(\mathrm{OH})$ ?

P. Schike: No. I think the Caselli/Herbst model which explained the equivalent scenario in Orion will not work in W3, since here the nitrogen bearing species are absent from the hottest spot in the hot core.

P. Caselli: In our model of the Orion Hot Core and Compact Ridge we found that differences in thermal history during the gravitational collapse of the two dense regions can lead to the observed chemical differentiation. In particular, the higher temperature in the Hot Core prevents the adsorption of the volatile $\mathrm{CO}$ molecule onto dust grains and thus $\mathrm{CH}_{3} \mathrm{OH}$ and $\mathrm{H}_{2} \mathrm{O}$ are not efficiently produced by surface hydrogenation reactions. On the other hand, complex carbonand nitrogen-bearing molecules can be formed through the adsorption of heavier species such as $\mathrm{C}_{2} \mathrm{~N}, \mathrm{C}_{3}, \mathrm{C}_{3} \mathrm{H}$, followed by addition reactions with atomic carbon and nitrogen and hydrogenation reactions. So, initial conditions (temperature, density, and fraction of gaseous atomic hydrogen) during the gravitational collapse phase are crucial for the formation of nitrogen-bearing species onto dust grain surfaces. We should re-run our models using the physical parameters derived for the $\mathrm{W} 3\left(\mathrm{H}_{2} \mathrm{O}\right)$ region and modified rates, to see if we can reproduce the observed abundances of $\mathrm{N}$-bearing species in this hot core. 


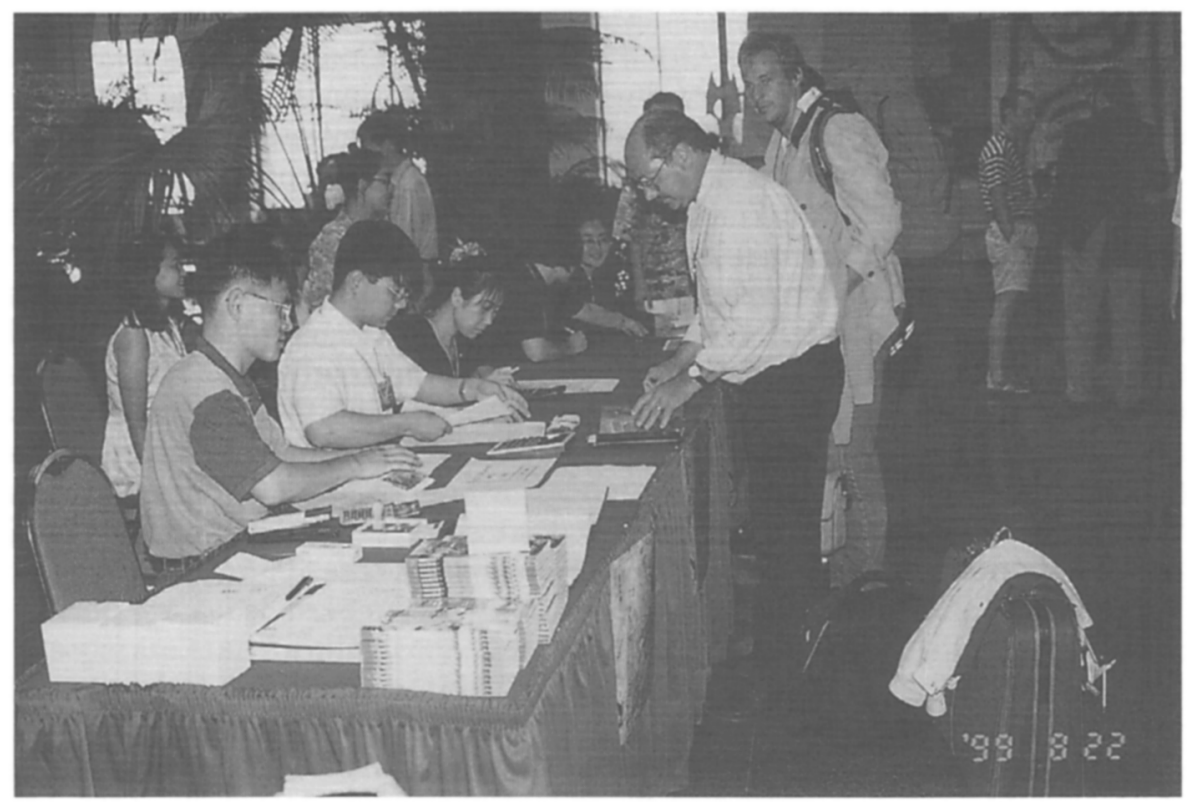

